\title{
Organischer Kohlenstoff in Bächen - Auswirkungen ackerbaulicher Bewirtschaftungspraktiken
}

\author{
Gabriele Weigelhofer (D) Alexandra Tiefenbacher $(\mathrm{D}) \cdot$ Martin Brandl (D) Peter Strauss $(\mathrm{D}$
}

Online publiziert: 25. November 2019

(c) Der/die Autor(en) 2019

\begin{abstract}
Zusammenfassung Mit über $45 \%$ Flächenanteil stellt die Landwirtschaft die dominante Landnutzungsform in Niederösterreich dar. Durch landwirtschaftliche Nutzung werden große Mengen an gelöstem organischem Material in Bäche eingebracht. Dieses organische Material führt zu einer Veränderung von Gewässerprozessen und beeinträchtigt den ökologischen Zustand der Gewässer. Das Ziel dieser Studie war es, die Auswirkungen verschiedener Bewirtschaftungspraktiken auf die Menge und die Qualität des gelösten organischen Materials in Fließgewässern zu untersuchen und die Folgen dieser Einträge auf den aquatischen Kohlenstoffumsatz und den Sauerstoffverbrauch abzuklären.
\end{abstract}

\author{
Mag. Dr. G. Weigelhofer \\ Department für \\ Wasser-Atmosphäre-Umwelt, \\ Institut für Hydrobiologie \\ und Gewässermanagement, \\ Universität für Bodenkultur Wien, \\ Gregor-Mendel-Straße 33, 1180 Wien, \\ Österreich
}

WasserCluster Lunz,

Dr.-Carl-Kupelwieser-Promenade 5,

3293 Lunz/See, Österreich

gabriele.weigelhofer@wcl.ac.at

DI A. Tiefenbacher · DI Dr. P. Strauss Institut für Kulturtechnik und Bodenwasserhaushalt, Bundesamt für Wasserwirtschaft, Pollnbergstraße 1, 3252 Petzenkirchen, Österreich

DI A. Tiefenbacher

alexandra.tiefenbacher@baw.at

DI Dr. P. Strauss

peter.strauss@baw.at

DI Dr. M. Brandl

Zentrum für Wasser-

und Umweltsensorik,

Donau-Universität Krems,

Dr.-Karl-Dorrek-Straße 30, 3500 Krems,

Österreich

martin.brandl@donau-uni.ac.at
Im Sommer 2017 und 2018 wurden Wasserproben aus Bächen mit unterschiedlicher Umlandnutzung, Kläranlagenabflüssen und der Donau in Niederösterreich entnommen und die Konzentrationen an gelöstem organischem Kohlenstoff (DOC) und Nährstoffen analysiert. Weiters wurde in kontrollierten Laborversuchen untersucht, wie sich Extrakte aus unterschiedlichen organischen Quellen, wie Wald- und Ackerboden, Blättern und Gülle, auf die mikrobielle Atmung in Bachsedimenten auswirken. Im Rahmen von Perkolationsversuchen analysierten wir die Auswirkungen von Düngung auf die Konzentrationen von organischem Material im Porenwasser unterschiedlicher Bodentypen.

Die Freilanderhebungen zeigen, dass die Konzentrationen an gelöstem organischen Kohlenstoff und Nährstoffen mit zunehmender Nutzung des Umlands durch Landwirtschaft oder Siedlungen ansteigen. Zudem kam es zu einer Anreicherung an labilen organischen Stoffen, was auf eine Belastung durch Mikroorganismen und auf erhöhte mikrobielle Umsatzraten in den Gewässern hindeutet. In den Laborversuchen führten manche DOC-Quellen, wie z.B. Gülle- oder Kuhfladenextrakt, zu einer signifikanten Erhöhung des bakteriellen Sauerstoffverbrauchs. Leicht verfügbare Kohlenstoffquellen haben somit das Potenzial, den Sauerstoffverbrauch in Gewässern zu stimulieren, wobei deren Wirkung sowohl von der Menge und Art der Kohlenstoffquelle als auch von der Beschaffenheit und der stofflichen Vorbelastung der Sedimente abhängt. In den Perkolationsversuchen führte eine nitratbasierende Düngung, unabhängig von der Art des Düngers (mineralisch oder organisch) zu einer Reduktion der DOCKonzentrationen im Sickerwasser. Der Grund für diese Reduktion könnte in einer Stimulation der Mineralisierung von organischem Material im Boden durch das eingebrachte Nitrat liegen. Unsere Studie weist auf die Bedeutung einer ausreichend langen Bodenpassage hin, um Fließgewässer in landwirtschaftlichen Regionen vor dem Eintrag von labilem organischen Kohlenstoff zu schützen. Das ist gerade im Hinblick auf eine prognostizierte Anhäufung von Trockenheit und Starkregenereignissen in der Zukunft wichtig, die zu einem verstärkten Eintrag von Bodenmaterial in Oberflächengewässer führen kann.

Schlüsselwörter Gelöster organischer Kohlenstoff · Fließgewässer .

Wasserqualität - Sauerstoffverbrauch · Bodensickerwasser

Organic carbon in streams - effects of agricultural land use practices

Abstract Agriculture is the dominant land use form in Lower Austria, covering more than $45 \%$ of the total area. Agriculture delivers significant amounts of dissolved organic carbon (DOC) to our streams, changing basic metabolic processes at the water-sediment interface and affecting the ecological state and the health of the aquatic systems. The aim of this study was to investigate the effects of agricultural practices on the amount and the quality of dissolved organic carbon discharged into streams and to clarify the consequences of these inputs onto the cycling of aquatic carbon and the oxygen consumption within the aquatic systems.

In summer 2017 and 2018, we took water samples from streams with different land use, wastewater effluents, and the Danube and analyzed them for concentrations of dissolved organic carbon and nutrients. In laboratory experiments, we studied the effects of leachates from different organic sources, such as forest soil, cropland soil, leaves, and manure on the microbial respiration in stream sediments. In addition, we performed percolation experiments to investigate the effects of fertilization on the concentration of 
dissolved organic matter leached from different soil types.

The concentrations of both dissolved organic carbon and nutrients increased with increasing agricultural and urban land use. In addition, we observed an enrichment with labile organic components, indicating increased pollution by microbes and elevated microbial turnover rates. Labile organic matter sources, such as e.g. manure leachate, significantly increased the microbial oxygen consumption in the sediments. However, the potential of stimulating microbial respiration depends on the amount and quality of the organic carbon sources as well as on the structure and the pollution of the sediments. In our percolation experiments, the application of nitrate-based fertilizers, whether mineral or organic, significantly decreased the amount of DOC in soil pore water. We assume that this reduction was caused by an increased mineralization of the organic matter in the soil, stimulated by the imported nitrate. Our study highlights the significance of a sufficiently long soil passage to protect streams in agricultural regions from excessive inputs of labile organic carbon. This factor gains in importance in the face of the predicted increase of long dry periods and heavy rain events in the future, which will increase the input of soil material into surface waters.

Keywords Dissolved organic carbon . Streams · Water quality · Oxygen consumption . Soil pore water

\section{Einleitung}

Mit über $45 \%$ Flächenanteil stellt Landwirtschaft die dominante Landnutzungsform in Niederösterreich dar. Die intensive landwirtschaftliche Nutzung von Einzugsgebieten kann zu einem beträchtlichen Eintrag an gelöstem organischem Kohlenstoff (DOC) und anorganischen Nährstoffen in Gewässersysteme führen, die grundlegende Stoffwechselprozesse verändern und den ökologischen Zustand von Fließgewässern langfristig verschlechtern (Graeber et al. 2015; Williams et al. 2010; Wilson and Xenopoulos 2009). Die Menge und Zusammensetzung des eingetragenen DOC kontrolliert das Wachstum und die Aktivität von Bakterien im Gewässer und beeinflusst den aquatischen Kohlenstoffkreislauf (Stanley et al. 2012; Tank et al. 2010).

DOC ist eine Mischung verschiedener organischer Kohlenstoffverbindungen, die je nach Herkunft und Alter aus einfachen Kohlenhydraten und Aminosäuren bis hin zu komplexen Molekülen mit teilweise hoher Aromatizität beste- hen (Graeber et al. 2015). Während kleine Moleküle, wie z.B. Einfachzucker, von Mikroorganismen sofort aufgenommen und größtenteils veratmet werden, müssen komplexe aromatische Gruppen erst nach und nach mithilfe von Enzymen aufgeschlossen werden und dienen zum Aufbau mikrobieller Biomasse (Findlay et al. 2001). Somit wirkt sich der Anteil an labilen und refraktären Kohlenstoffverbindungen auf den Stoffwechsel von Gewässerbakterien aus. In natürlichen oder naturnahen Bächen mit einer hydrologisch gut angebundenen Bachaue stammt ein Großteil des im Wasser gelösten organischen Materials von Ufergehölzen und weist daher einen hohen Anteil an refraktären Bestandteilen auf (Stutter et al. 2018). Fehlt jedoch die Beschattung, kann es in nährstoffbelasteten Gewässern zu einem massiven Wachstum an Algen kommen, deren Exsudate zu einer Erhöhung des labilen Kohlenstoffs führen. Weitere Quellen für labilen Kohlenstoff in landwirtschaftlichen Gebieten sind unter anderem Ausbringung von Gülle, Drainagen oder Bodenbearbeitung. Hohe Mengen an leicht verfügbarem Kohlenstoff können zu einer erhöhten Sauerstoffzehrung in den Sedimenten von Bächen führen, die sich unter ungünstigen Bedingungen, wie z.B. einer geringen Wasserführung und hohen Wassertemperatu-

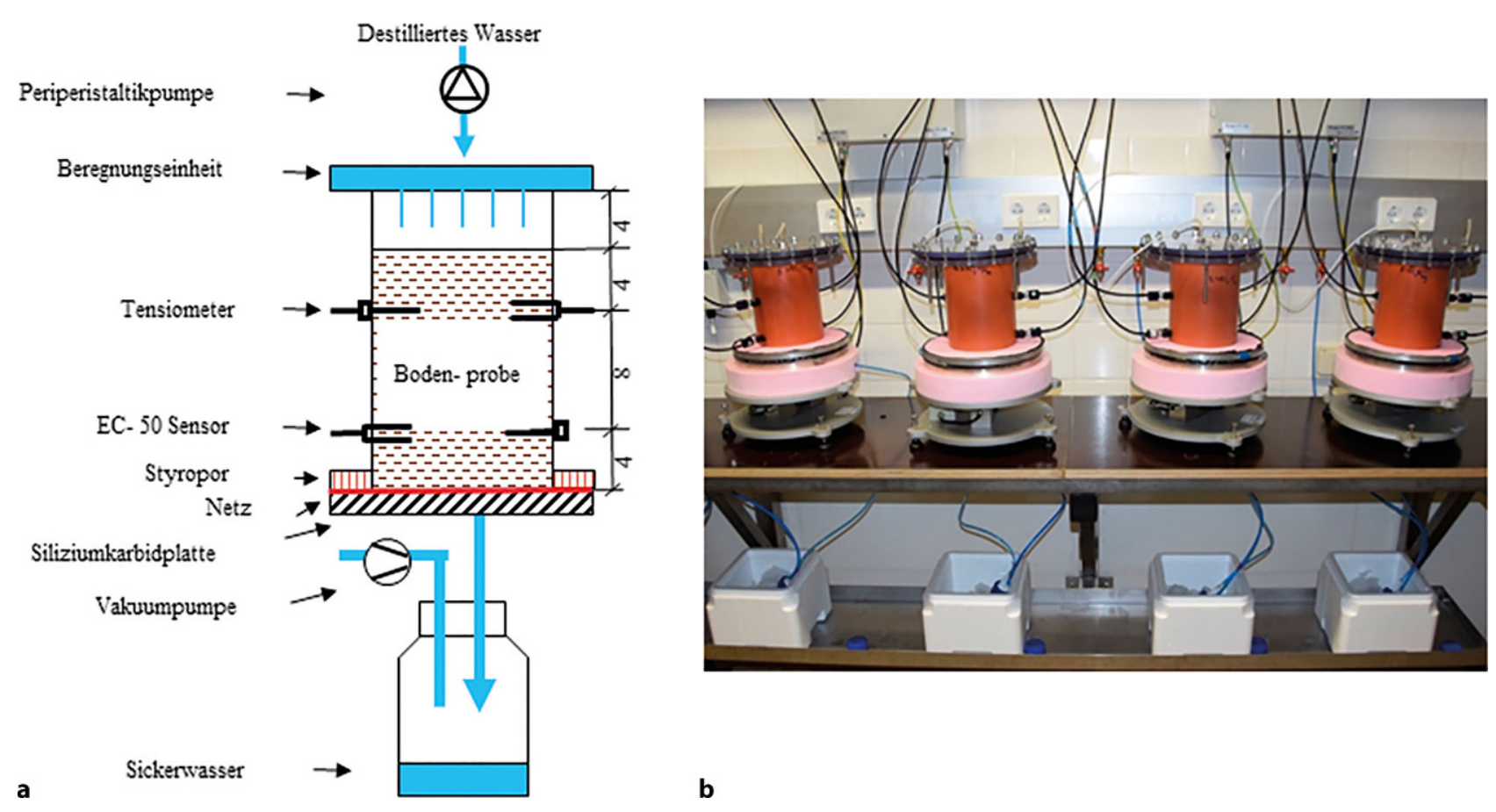

Abb. 1 Perkolationsversuch; a schematische Darstellung einer Einheit; b Teil der Anlage 


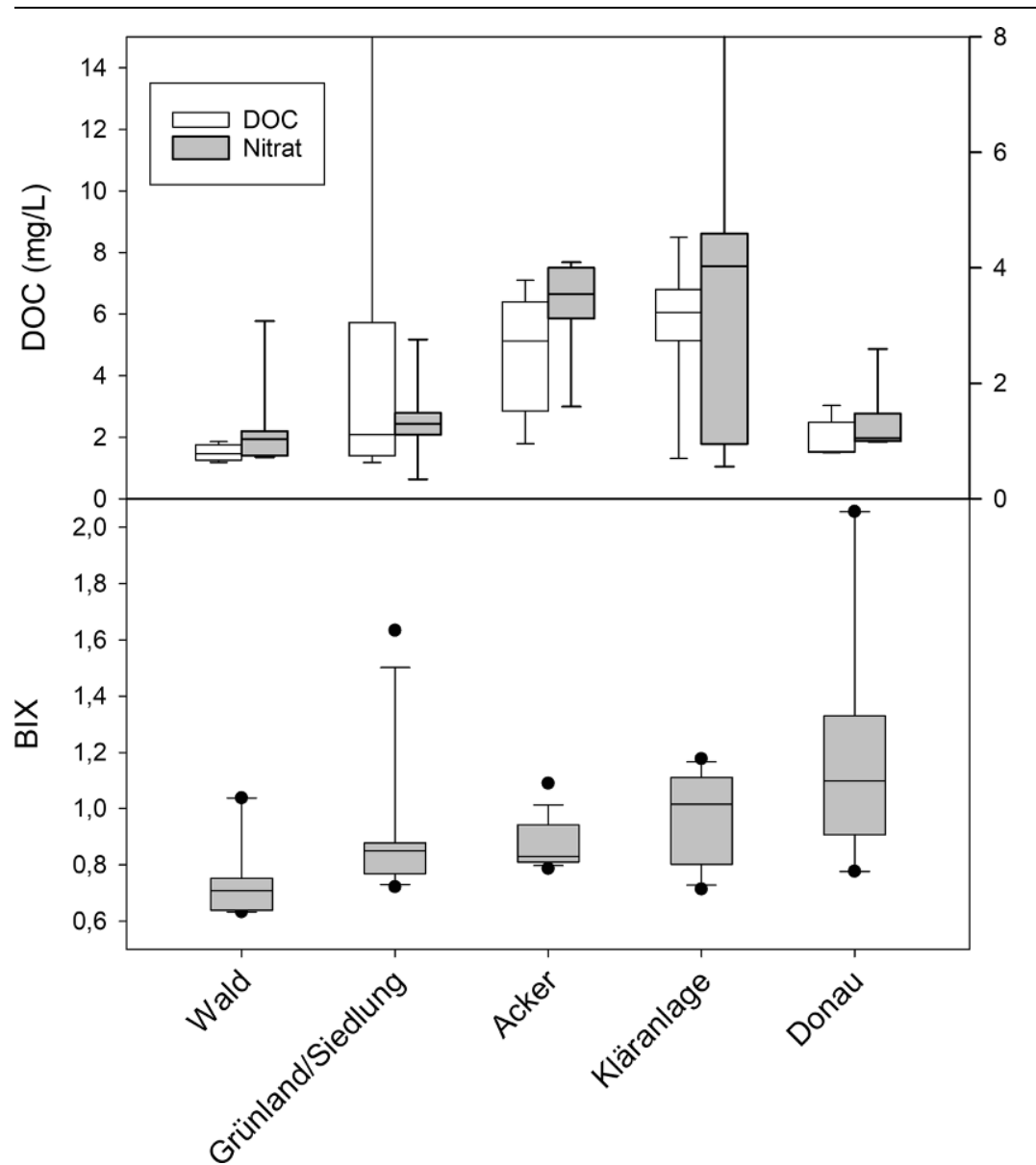

Abb. 2 DOC-Konzentrationen, Nitratkonzentrationen und Frische-Index BIX (Anzeiger für mikrobiell erzeugtes DOM) in niederösterreichischen Bächen mit unterschiedlicher Landnutzung (Wald, intensiv bewirtschaftetes Grünland und/oder Siedlungen, Äcker), Kläranlagenabflüssen und entlang der Donau zwischen Hainburg und Ybbs (10-, 25-, 50-, 75- und 90-\%-Perzentile; $n=5-15$ )

ren, bis in die Wassersäule bemerkbar macht. Trotz einer steigenden Anzahl an Studien über den Einfluss von landwirtschaftlicher Nutzung auf die Menge und die Qualität des organischen Materials in Bächen ist bis heute der Zusammenhang zwischen bestimmten Bewirtschaftungspraktiken, der Veränderung des organischen Materials beim Transport durch den Boden und dessen Einfluss auf die Wasserqualität nicht ausreichend bekannt. Das NFB-Projekt „Organic carbon cycling in streams: Effects of agricultural land use (ORCA)" (www.organic-carbon.at) beschäftigte sich mit dem Einfluss landwirtschaftlicher Bewirtschaftungspraktiken (z.B. Düngung) und hydrologischer Transportpfade (u.a. Oberflächenabfluss, Grundwasser, Drainagen etc.) auf die Menge und die Zusammensetzung des gelösten organischen Materials in Fließgewässern. Des Weiteren untersuchten wir, wie sich unterschiedliche DOCQuellen auf die Wasserqualität und den Sauerstoffhaushalt der Gewässer auswirken.

\section{Methoden}

\subsection{Gewässerscreening}

Im Sommer 2017 und 2018 wurden Wasserproben aus Bächen mit unterschiedlicher Umlandnutzung, Kläranlagenabflüssen und der Donau in Niederösterreich entnommen und die Konzentrationen an anorganischen Nährstoffen (photometrisch) und gelöstem organischem Kohlenstoff (gaschromatographisch mit Sievers*900 portable TOC-Analyzer) analysiert. Zur Bestimmung der qualitativen Zusammensetzung des DOC wurden Fluoreszenzund Absorptionsspektren mit einem UV-VIS-Spectrometer und einem $\mathrm{Hi}$ - tachi F-7000 Fluoreszenzspektrometer über einen Wellenlängenbereich von 200 bis $600 \mathrm{~nm}$ gemessen (Coble 1996). Mittels des R-Packages staRdom wurden verschiedenen Indizes errechnet, die die Qualität des DOC beschreiben. So weisen der Frische-Index (BIX) und der Fluoreszenz-Index (FIX) auf organisches Material hin, das voraussichtlich frisch von Mikroorganismen produziert wurde. Im Gegensatz dazu steigt der Humifizierungsindex (HIX) an, wenn das Material einen hohen Humifizierungsgrad aufweist (Baker and Spencer 2004; Coble 1996).

\subsection{Sauerstoffzehrung in Bachsedimenten}

Die Sauerstoffzehrung in Bachsedimenten wurde in luftdichten Flaschen im Dunkeln bei Raumtemperatur über einen Zeitraum von $24 \mathrm{~h}$ ermittelt. Da$\mathrm{zu}$ wurden Feinsedimente mit einem Korndurchmesser $<4 \mathrm{~mm}$ aus drei unterschiedlich belasteten Bächen (Gamingbach oberhalb Gaming, Sierning unterhalb Kilb, Seitengraben in Petzenkirchen) entnommen und nach Zugabe verschiedener organischer Extrakte in nährstoff- und kohlenstoffarmen Brunnenwasser inkubiert. Der Sauerstoffverbrauch wurde über kabellose Sauerstoffsensoren (PreSense) in 2-stündigen Intervallen gemessen. Die Extrakte wurden durch Kaltauszug über Nacht aus folgenden Quellen gewonnen: Ackerboden, Waldboden, Laubblätter, Gülle und Kuhfladen. Die Zugabe der Extrakte wurde so berechnet, dass $\mathrm{zu}$ Beginn des Versuchs ungefähr dieselben Endkonzentrationen an DOC in allen Flaschen vorhanden waren. Die Berechnung der Sauerstoffzehrung erfolgte mittels linearer Regression der Sauerstoffabnahme gegen die Zeit.

\subsection{Perkolationsversuche}

Um die Wirkung der landwirtschaftlichen Intensivierung auf die Aktivierung von DOC zu überprüfen, wurden kontrollierte Perkolationsversuche durchgeführt. Dabei wurden ungestörte Bodenprofile zweier Böden mit unterschiedlichen Textureigenschaften (schluffiger Lehm und lehmiger Sand) in die Mikrolysimeteranlage des Instituts für Kulturtechnik und Bodenwasserhaushalt eingebaut. In einem 45-tägigen Beregnungsversuch wurde die Auswirkung unterschiedlicher Düngungsmaßnahmen auf die Mobilisie- 


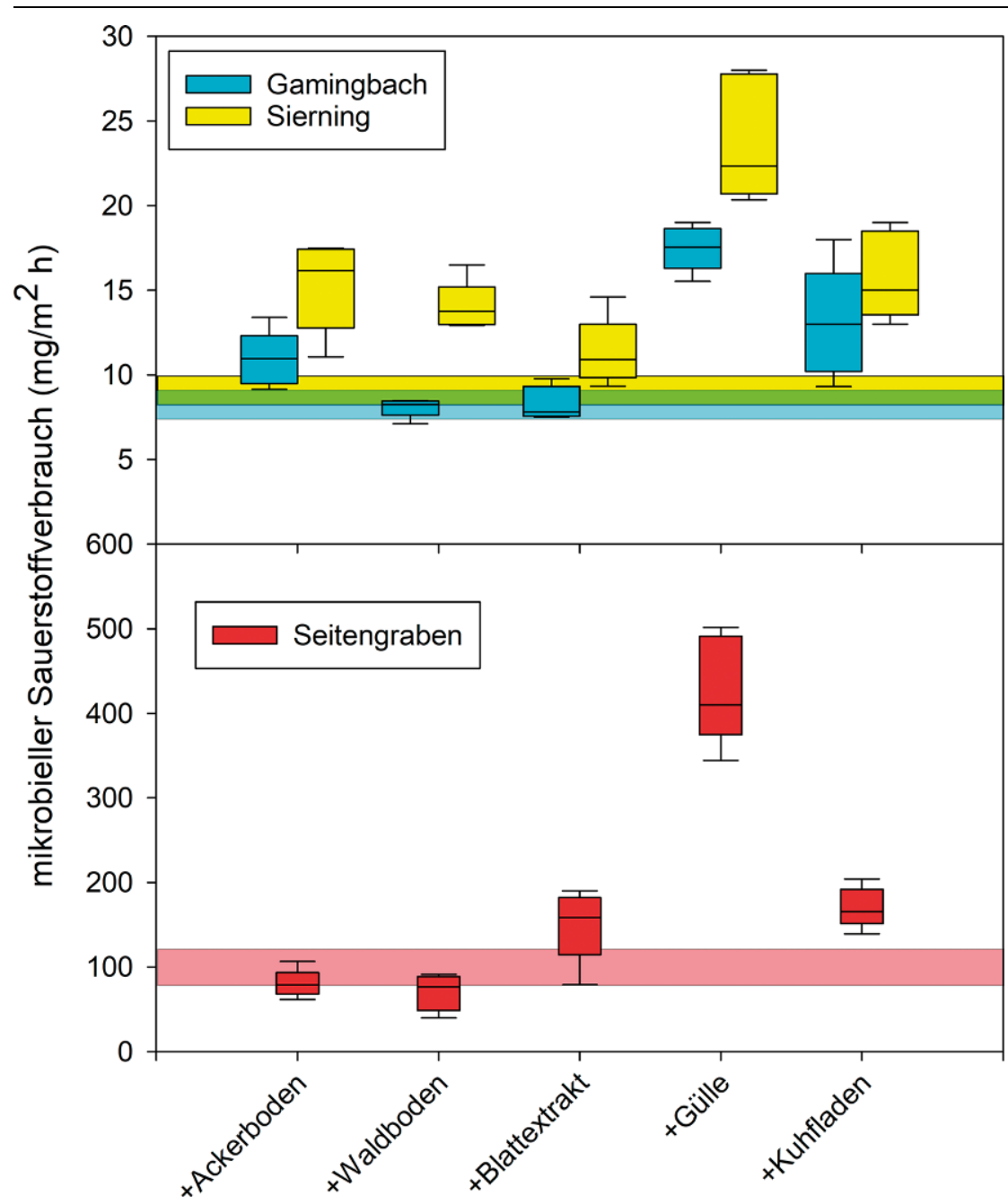

Abb. 3 Mikrobieller Sauerstoffverbrauch in den Sedimenten von drei Bächen nach der Zugabeverschiedener Extrakte (10-, 25-, 50-,75- und 90-\%-Perzentile; $n=5)$. Die größte Wirkung auf den Sauerstoffverbrauch hatte der Gülleextrakt. Die horizontalen Balken zeigen den Grundsauerstoffverbrauch ohne Extrakt

rung und Auswaschung von gelöstem organischen Kohlenstoff untersucht. Die untersuchten Düngungsvarianten waren eine organische Düngung mit Schweinegülle und eine mineralische Düngung mit Calciumammoniumnitrat (NAC 27). Die applizierte Stickstoffkonzentration von $130 \mathrm{~kg} \mathrm{~N} \cdot \mathrm{ha}^{-1}$ entspricht einer in Österreich üblichen Düngemenge für Mais und Weizen (Richtlinien für die sachgerechte Düngung 2017). Nach der Düngung wurde im sechstägigen Rhythmus sowohl die Konzentration als auch die Qualität des gelösten organischen Kohlenstoffs im Sickerwasser der Böden bestimmt. Abb. 1 zeigt die schematische Darstellung des Versuchsaufbaus.

\section{Ergebnisse und Diskussion}

\subsection{Menge und Qualität von DOC in} niederösterreichischen Gewässern

Die Konzentrationen an gelöstem organischen Kohlenstoff steigen mit zunehmender Nutzung des Umlands durch Landwirtschaft oder Siedlungen an und erreichen im Sommer teilweise Werte, die auch in Kläranlagenabflüssen zu finden sind (Abb. 2). Ähnlich ansteigende Konzentrationen von Wald zu Ackerbaugebieten sind auch bei den gelösten Nährstoffen, wie z.B. Nitrat, zu beobachten. Im Gegensatz dazu weist die Donau vergleichsweise geringe DOCund Nitratkonzentrationen auf, was hauptsächlich auf Verdünnungseffekte zurückzuführen ist. Derartige Verdünnungseffekte sind normalerweise auch bei kleineren Fließgewässern in Zeiten erhöhter Wasserstände zu beobachten. So betragen z. B. die Nitrat- und DOCKonzentrationen im Frühjahr nach der Schneeschmelze häufig weniger als $50 \%$ der Sommerwerte. Die Ergebnisse zeigen außerdem, dass eine intensive Nutzung des Einzugsgebiets sich nicht nur in den Konzentrationen, sondern auch in der Qualität des eingetragenen organischen Materials auswirkt. Sowohl der Frische-Index BIX als auch der Fluoreszenz-Index FIX (Anzeiger für frisch produziertes Material mikrobiellen Ursprungs; Abb. 2) steigen von den Waldbächen zu den Bächen in intensiv genutztem Ackerland an, was auf eine Belastung durch Mikroorganismen und auf hohe mikrobielle Umsatzraten in den Gewässern hindeutet. Die höchsten Werte sind, wie erwartet, in Kläranlagenabflüssen, aber auch in der Donau zu finden.

\subsection{Einfluss verschiedener DOC- Quellen auf die Sauerstoffzehrung in Bachsedimenten}

Die Sauerstoffzehrung in den Sedimenten hängt u. a. vom organischen Gehalt, dem Feinsedimentanteil und den Nährstoffgehalten in den Sedimenten ab. Der Eintrag von erodiertem Boden aus landwirtschaftlich genutzten Flächen in kleine Bäche kann hier bereits ohne zusätzliche DOC-Quellen zu einer Erhöhung des Grundumsatzes führen. So wies der Seitengraben in Petzenkirchen aufgrund der höheren organischen $\mathrm{Ge}$ halte und Feinsedimentanteile einen 10-fach höheren Grundsauerstoffverbrauch auf als der Gamingbach und die Sierning (Abb. 3, horizontale Balken). Die ursprünglichen DOC-Konzentrationen im Brunnenwasser lagen bei $0,4 \mathrm{mg} / \mathrm{L}$ und konnten durch die $\mathrm{Zu}$ gabe der Extrakte auf 2,2 mg/L (Ackerboden) bis $4,3 \mathrm{mg} / \mathrm{L}$ (Gülle) angehoben werden. Die größte Wirkung auf den bakteriellen Sauerstoffverbrauch hatte der Gülleextrakt, der in allen Sedimenten zu einer signifikanten Erhöhung auf das mindestens 3-Fache des Grundverbrauchs führte (Abb. 3). Ebenso führten die Extrakte von Kuhfladen und Ackerboden zu einer Stimulation der mikrobiellen Atmung. Von den Gewässern reagierten die Sedimente der Sierning am deutlichsten auf die Zufuhr von DOC. Sie zeigten eine signifikante Erhöhung des Sauerstoffverbrauchs bei fast allen DOC-Quellen, was auf eine mögliche Kohlenstofflimitierung 


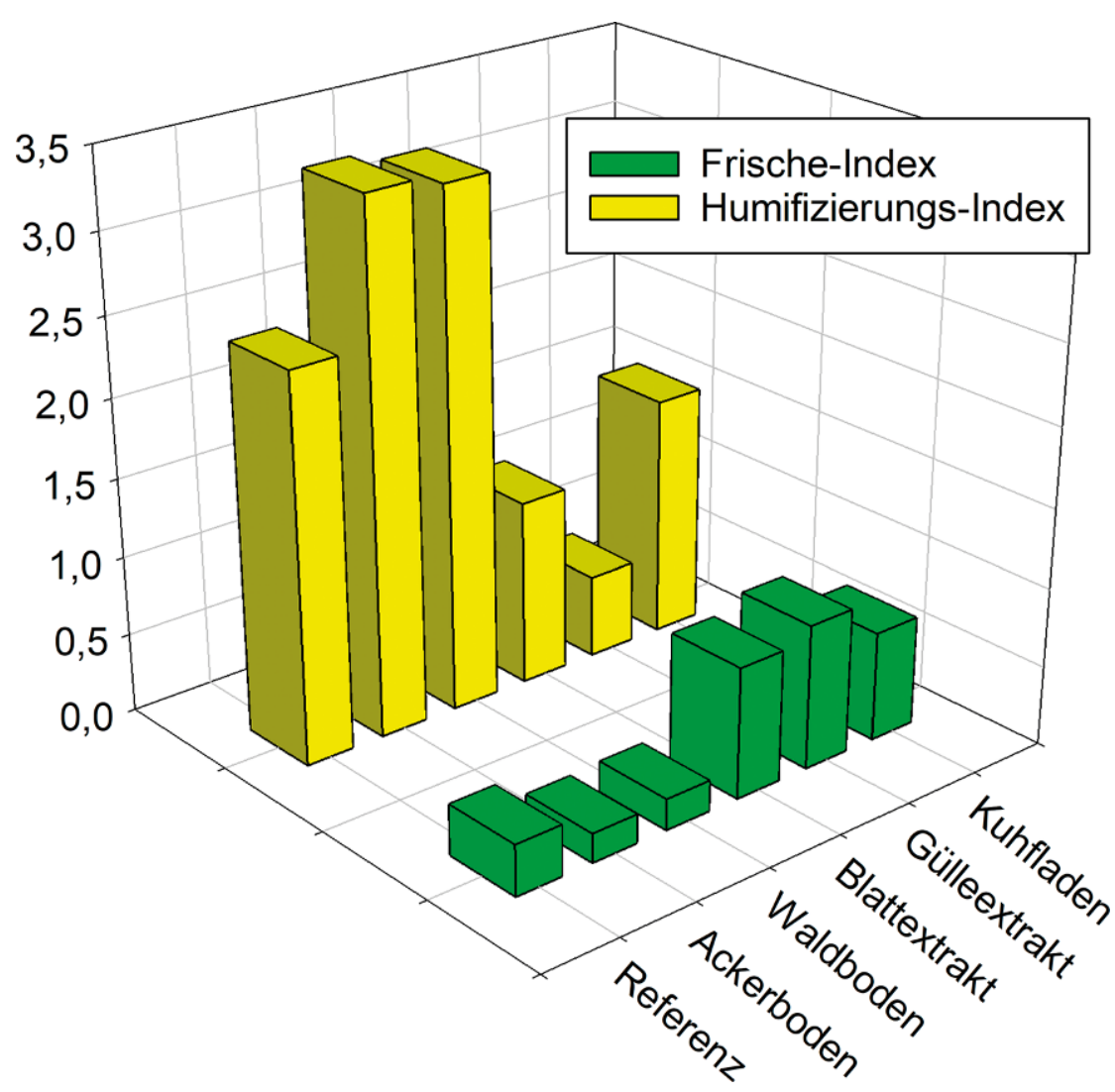

Abb. 4 Frische-Index und Humifizierungs-Index der Extrakte und des Bachwassers zu Beginn der Experimente. Die Extrakte der Blätter, Gülle und Kuhfladen wiesen einen hohen Anteil an labilen Kohlenstoffverbindungen auf

hindeutet. Derartige Kohlenstofflimitierungen konnten bereits bei anderen Bächen in landwirtschaftlichen Regionen beobachtet werden (Stutter et al. 2018). In diesen Fällen sind die Mengen an verfügbarem Kohlenstoff im Vergleich zu den Mengen an Stickstoff und Phosphor gering und liegen unter dem idealen stöchiometrischen C:N:P-Verhältnis, wodurch der Kohlenstoff zum limitierenden Faktor für eine mikrobielle Nährstoffaufnahme wird.

Für die Aufnahme und die Veratmung von DOC durch Mikroorganismen sind neben der Menge an Kohlenstoff auch die Anteile an labilen Kohlenstoffverbindungen ausschlaggebend. Die Extrakte von Acker- und Waldboden zeigten einen niedrigen Frische-Index (BIX) und einen hohen Humifizierungs-Index (HIX), was auf überwiegend refraktäre Bestandteile hinweist (Abb. 4). Im Gegensatz dazu wiesen die Extrakte der Laubblätter, Gülle und Kuhfladen zu Beginn einen hohen Frische-Index und einen niedrigen Humifizierungs-Index auf, was auf eine leichte Verfügbarkeit dieser DOC-
Sickerwasser, wobei in mineralisch gedüngten Boden die niedrigsten und in der ungedüngten Kontrolle die höchsten DOC-Konzentrationen gemessen wurden (Abb. 5). Die Nitratkonzentrationen im Sickerwasser wurden hingegen durch die Düngung erhöht. Dementsprechend wurden die höchsten Nitratkonzentrationen in mineralisch gedüngten Böden gemessen und die zweithöchsten Werte konnten mit der organischen Düngung erzielt werden. Dem nachstehenden Streudiagramm ist zu entnehmen, dass im ungedüngten Boden die DOC-Konzentration im Sickerwasser proportional zur Nitratkonzentration ansteigt (Abb. 5). Im Gegensatz dazu verhält sich die Beziehung zwischen der DOC-Konzentration und dem Nitrat im Sickerwasser von gedüngten Böden umgekehrt proportional. Aus diesen Ergebnissen können wir schließen, dass eine nitratbasierende Düngung die Mineralisierung des organischen Materials im Boden ankurbelt, welche $\mathrm{zu}$ einer Verminderung der DOC-Konzentrationen im Sickerwasser führte. Diese These kann durch den erhöhten Anteil von mikrobiell produziertem organischen Material im Sickerwasser der gedüngten Böden unterstrichen werden (Daten nicht angezeigt). Auch in der Literatur finden sich Belege, dass eine Düngung die DOC-Konzentration im Sickerwasser aufgrund einer Stimulierung der Mineralisationsprozesse im Boden durch die zugefügten Nährstoffe reduzieren kann (Lu et al. 2013).

\section{Schlussfolgerungen} zusätzlich Phosphor einbrachte. Die Ergebnisse zeigen, dass leicht verfügbare Kohlenstoffquellen das Potenzial haben, den Sauerstoffverbrauch in Gewässern zu stimulieren, wobei deren Wirkung sowohl von der Menge und Art der Kohlenstoffquelle als auch von der Beschaffenheit und der stofflichen Belastung der Sedimente abhängt.

\subsection{Perkolationsversuche}

Sowohl die Art als auch die Menge der Düngung landwirtschaftlicher Böden beeinflusst die DOC-Konzentration im Sickerwasser (Bolan et al. 2011; Cleveland et al. 2004). In unseren Perkolationsversuchen bewirkten beide Düngervarianten, sowohl die mineralische (NAC, Mononitratdünger) als auch die organische Düngung (Schweinegülle), eine Konzentrationsminderung des gelösten organischen Kohlenstoffs im
Die intensive landwirtschaftliche Nutzung der Einzugsgebiete kann zu einem erhöhten Eintrag an labilem gelösten organischen Kohlenstoff in Fließgewässer führen. Diese labilen Kohlenstoffquellen haben das Potenzial, den Sauerstoffverbrauch in den Gewässern zu stimulieren und zu einer verstärkten Sauerstoffzehrung in den Sedimenten $\mathrm{zu}$ führen. Erhöhte Wassertemperaturen, Nährstoffbelastungen sowie eine Anreicherung an organischem und anorganischem Feinmaterial in den Bachsedimenten aufgrund erhöhter Bodenerosion können diesen Effekt noch weiter verstärken. Unsere Perkolationsversuche zeigen, dass bei entsprechend langer Bodenpassage die Wirkung von Düngung auf den Eintrag von organischem Kohlenstoff und Nährstoffen 


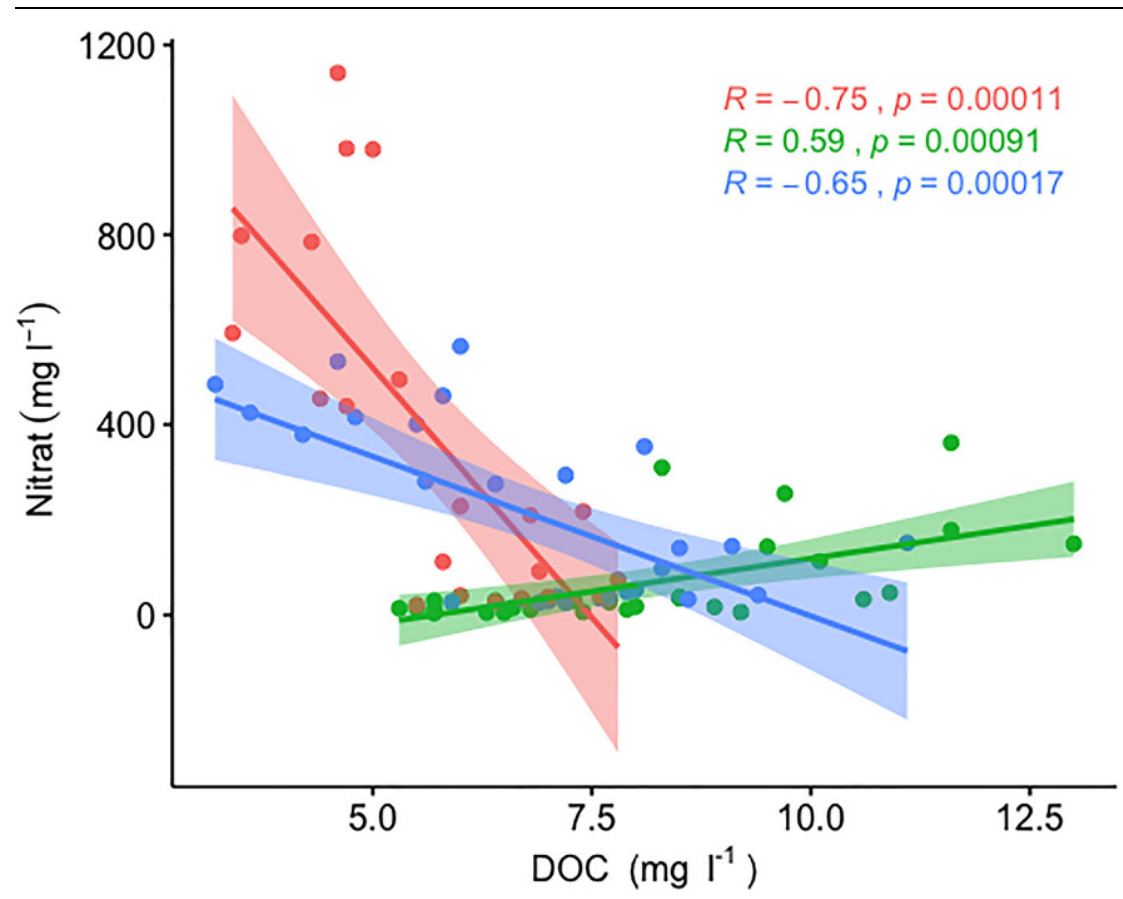

Abb. 5 Streudiagramm des Nitratgehalts und des gelösten organischen Kohlenstoffgehalts im Sickerwasser. Regressionsgerade und Konfidenzintervall $(0,95)$ der Düngevarianten wird gezeigt. Kontrolle (grün), mineralische (NAC, blau) und organische Düngung (Gülle, rot)

in Fließgewässer jedoch abgemildert werden kann.

Auf globaler Ebene hat die Intensivierung der Landwirtschaft bereits den Eintrag von gelöstem organischen Kohlenstoff in Oberflächengewässer verändert. Zusätzlich werden durch die Klimaerwärmung künftig viele Regionen während der Sommermonate mit trockenen Böden und Starkregen zu kämpfen haben. Besonders Starkregenereignisse auf trockengefallenen Böden werden Oberflächenabflüsse mit hohen Sedimentkonzentrationen begünstigen. Folglich wird künftig mehr gelöster organischer Kohlenstoff aus dem Oberboden ohne jegliche Bodenpassage in anliegende Gewässer verfrachtet werden. Daher ist es un- relativ einfach und rasch vor Ort erhoben und so eine mögliche Belastung durch labiles, mikrobiell produziertes organisches Material festgestellt werden kann (Posnicek et al. 2018).

Danksagung Dieses Projekt wurde vom Land Niederösterreich im Rahmen des Science Calls 2015 durch die NÖ Forschungs- und Bildungsges.m.b.H. (SC15-002) gefördert. Die Analysen der Wasserproben wurden durch eine Studierende im Rahmen ihres FEMTec-Praktikums sowie durch SchülerInnen des Josephinums Wieselburg im Rahmen ihres Ferialpraktikums mit finanzieller Unterstützung der Österreichischen Forschungsförderungsgesellschaft (FFG) durchgeführt. Die Freilanderhebungen und Laboranalysen wurden von Beate Pitzl, Annette Puritscher, Gertraud Stenicka, Claudia Dienstl und Elena Pfeiffer durchgeführt. Ein Teil des Gewässermonitorings wurde in Zusammenarbeit mit CEST, Philipp Fruhmann, im Rahmen des NFBProjekts „Fast and selective detection of organic pollutants in water" (SC15004) durchgeführt.

Funding Open access funding provided by University of Natural Resources and Life Sciences Vienna (BOKU). die Wirkung von landwirtschaftlicher Bewirtschaftung auf den Austrag von gelöstem organischen Kohlenstoff aus dem Boden in die Oberflächengewässer abschätzt. Zudem wäre zu empfehlen, die Qualität des gelösten organischen Materials mittels einfacher Indizes in ein Gewässermonitoring aufzunehmen. Das könnte z.B. mithilfe von derzeit in Entwicklung befindlichen Sensoren geschehen, die diverse organische Belastungen von Gewässern anhand ausgewählter Fluoreszenz-Peaks direkt im Freiland messen können. So wurde auch im Rahmen des ORCA-Projekts ein Sensor entwickelt und getestet, mit dessen Hilfe der Frische-Index und der Fluoreszenz-Index in der Wassersäule

Open Access Dieser Artikel wird unter der Creative Commons Namensnennung 4.0 International Lizenz (http:// creativecommons.org/licenses/by/4. 0/deed.de) veröffentlicht, welche die Nutzung, Vervielfältigung, Bearbeitung, Verbreitung und Wiedergabe in jeglichem Medium und Format erlaubt, sofern Sie den/die ursprünglichen $\mathrm{Au}$ tor(en) und die Quelle ordnungsgemäß nennen, einen Link zur Creative Commons Lizenz beifügen und angeben, ob Änderungen vorgenommen wurden. .

Literatur

Baker, A., Spencer, R.G.M. (2004): Characterization of dissolved organic matter from source to sea using fluorescence and absorbance spectroscopy. Science of the Total Environment 333, 217-232.

Bolan, N.S., Adriano, D.C., Kunhikrishnan, A. James, T., McDowell, R., Senesi, N. (2011): Dissolved Organic Matter, in: Advances in Agronomy. Elsevier, pp. 1-75. https://doi.org/10.1016/ B978- 0-12-385531-2.00001-3

Cleveland, C.C., Neff, J.C., Townsend, A.R. Hood, E. (2004): Composition, Dynamics, and Fate of Leached Dissolved Organic Matter in
Terrestrial Ecosystems: Results from a Decomposition Experiment. Ecosystems 7. https://doi. org/10.1007/s10021-003-0236-7

Coble, P.G. (1996): Characterization of marine and terrestrial DOM in seawater using excitation-emission matrix spectroscopy. Marine Chemistry 51, 325-346.

Findlay, S., Quinn, J.M., Hickey, C.W., Burrell, G., Downes, M. (2001): Effects of land use and riparian flowpath on delivery of dissolved organic carbon to streams. Limnol. Oceangr. 46, 345-355. Graeber, D., Boëchat, I.G., Encina-Montoya, F., Esse, C., Gelbrecht, J., Goyenola, G., Gücker,
B., Heinz, M., Kronvang, B., Meerhoff, M., Nimptsch, J., Pusch, M.T., Silva, R.C.S., Schiller, D. von, Zwirnmann, E. (2015): Global effects of agriculture on fluvial dissolved organic matter. Scientific Reports 5, 16328. https://doi.org/10. 1038/srep 16328

Lu, X., Gilliam, F.S., Yu, G., Li, L., Mao, Q., Chen, H., Mo, J. (2013): Longterm nitrogen addition decreases carbon leaching in a nitrogen-rich forest ecosystem. Biogeosciences 10, 3931-3941. https://doi.org/10.5194/bg-10-3931-2013 Posnicek, T., Weigelhofer, G; Eder A., Brandl, M. (2018): Highly Integrated and Mobile Sensor Sys- 
tem for Dissolved Organic Matter in Stream Ecosystems. MDPI Proceedings 2018, 2(13): 1507 Stanley, E.H., Powers, S.M., Lottig, N.R., Buffam, I., Crawford, J.T. (2012): Contemporary changes in dissolved organic carbon ( DOC ) in human-dominated rivers: is there a role for DOC management? Freshwater Biology 57, 26-42. https://doi.org/10.1111/j.1365-2427. 2011.02613.x

Stutter, M.I., Graeber, D., Evans, C.D., Wade, A.J., Withers, P.J.A. (2018): Balancing macronutrient stoichiometry to alleviate eutrophication. Science of the Total Environment 634, 439-447. https://doi.org/10.1016/j.scitotenv.2018.03.298
Tank, J.L., Rosi-Marshall, E.J., Griffiths, N.A. Entrekin, S.A., Stephen, M.L. (2010): A review Entrekin, S.A., Stephen, M.L. (2010): A review
of allochthonous organic matter dynamics and metabolism in streams. Journal North American Benthological Society 29, 118-146. https://doi. org/10.1899/08-170.1

Williams, C.J., Yamashita, Y., Wilson, H.F., Jaffe, R., Jaffé, R., Xenopoulos, M. (2010): Unraveling the role of land use and microbial activity in shaping dissolved organic matter characteristics in stream ecosystems. Limnology and Oceanography 55, 1159-1171. https://doi.org/10.4319/ lo.2010.55.3.1159
Wilson, H.F., Xenopoulos, M. a. (2009): Effects of agricultural land use on the composition of fluvial dissolved organic matter. Nature Geoscience 2, 37-41. https://doi.org/10.1038/ngeo391

Hinweis des Verlags Der Verlag bleibt in Hinblick auf geografische Zuordnungen und Gebietsbezeichnungen in veröffentlichten Karten und Institutsadressen neutral. 